\title{
Anti-Candida activity of antimicrobial impregnated central venous catheters
}

L. Cobrado ${ }^{1,3^{*}+}$ (D) A. Silva-Dias ${ }^{1,3+}$, M. M. Azevedo ${ }^{1,3}$ and A. Rodrigues ${ }^{1,2,3}$

\begin{abstract}
Background: Whenever the rate of central line-associated bloodstream infections (CLABSIs) remains high even after the implementation of preventive strategies, the use of chlorhexidine/silver sulfadiazine (CSS) or minocycline/rifampin (MR)-impregnated central venous catheters (CVCs) is currently recommended. Nevertheless, the efficacy of such CVCs against Candida albicans and other emerging non-albicans spp. has been insufficiently studied. This study aims to compare the activity of CSS and MR-impregnated CVCs against the yeasts most frequently isolated from CLABSIs.

Methods: For biofilm formation assays, type strains and clinical isolates of C. albicans, C. glabrata and C. parapsilosis sensu stricto were used. Segments of standard polyurethane, MR and second-generation CSS-CVCs were tested. The biofilm metabolic activity was measured by a semi-quantitative XTT reduction assay.

Results: CSS catheter segments significantly reduced the biofilm metabolic activity by all tested Candida spp., with inhibition ranging from $60 \%$ to $100 \%$. The MR catheter segments promoted C. albicans and C. parapsilosis biofilm formation and exhibited an inconspicuous effect against C. glabrata.

Conclusions: Among the recommended antimicrobial CVCs, CSS-CVCs proved to be superior in the inhibition of biofilm formation by the most frequent yeasts causing CLABSIs. Data from this in vitro study may suggest that patients at high risk for invasive candidosis could benefit from the use of CSS-CVCs.
\end{abstract}

Keywords: Healthcare-associated infections, Antimicrobial central venous catheters, Candidemia

\section{Background}

Although there are some signs of a possible decline in the incidence of central line-associated bloodstream infections (CLABSIs) [1], it certainly remains a major medical concern for which further preventive measures can be taken to minimize its impact upon morbidity, increased length of hospital stay and financial resources expended. A 46\% decrease in the incidence has occurred in US hospitals from 2008 to 2013, even though an estimated 30,100 CLABSIs still occur each year [2]. As reported by the European Centre for Disease Prevention and Control, bloodstream infections may be catheterrelated in $43.3 \%$ of cases, with a mean device-adjusted rate in patients staying in the Intensive Care Unit (ICU)

\footnotetext{
* Correspondence: Icobrado@med.up.pt

${ }^{\dagger}$ Equal contributors

'Division of Microbiology, Department of Pathology, Faculty of Medicine, University of Porto, Alameda Prof. Hernâni Monteiro, 4200 Porto, Portugal ${ }^{3}$ CINTESIS, Center for Health Technology and Services Research, Faculty of Medicine, University of Porto, Porto, Portugal

Full list of author information is available at the end of the article
}

for more than 2 days of 3.0 CLABSI episodes per 1000 CVC-days. Among isolated microorganims in ICUacquired bloodstream infections from participant EU countries, Candida spp. represented a total of 8.2\% [3].

Globally, Candida spp. are the fourth frequent causative pathogen of CLABSIs [4] and contribute to $12 \%$ of all CLABSIs [5], with an attributable mortality of $38 \%$ [6]. Through the colonization of the skin, manipulation of the catheter hub, contamination of infusates or from a distant focus of infection, Candida cells may adhere to the CVC surface and produce extracellular polymers, providing a structural matrix that will further facilitate adhesion [7]. In such biofilm, Candida cells display an increased resistance to antifungal agents and, from such strategic anchorage point, yeasts may disperse into the bloodstream, leading to serious infection [7-9].

Despite being a heterogeneous group of organisms, most of the invasive infections are caused by $C$. albicans, $C$. parapsilosis and C. glabrata [10, 11]. Risk factors for invasive candidosis include the presence of a CVC, prolonged 
ICU stay, use of broad-spectrum antibiotics, previous gastrointestinal surgery, neutropenia, diabetes mellitus, burns and administration of total parenteral nutrition $[12,13]$. Moreover, a trend towards infections by non-albicans spp. has been noticed in the past years [14-16]. This change in epidemiology can be associated with severe immunosuppression or illness, exposure to broad-spectrum antibiotics, prematurity or older age [14].

Recommendations for CLABSI prevention issued by the US Centers for Disease Control and Prevention (CDC) target, for example, the education of healthcare personnel, hand hygiene and aseptic techniques for insertion and care of central venous catheters (CVCs) [17]. Even though, whenever the rate of infection remains high even after successful implementation of a comprehensive strategy to reduce CLABSIs, the use of chlorhexidine/silver sulfadiazine (CSS) or minocycline/ rifampin (MR)-impregnated CVCs is currently recommended [17]. According to the respective manufacturer's technology sheet, second-generation CSS-CVCs (coated on both the external and internal surfaces) should exhibit antimicrobial activity against Klebsiella pneumoniae, Escherichia coli, Pseudomonas aeruginosa, Staphylococcus aureus, S. epidermidis and Candida albicans [18] and MRCVCs are expected to display a broad-spectrum protection against Gram-positive, Gram-negative and fungal infections [19]. In spite of this wide antimicrobial spectrum, the efficacy of impregnated CVCs against yeasts, particularly nonalbicans species, has not been fully addressed. Moreover, if effectiveness of MR-CVCs against Candida has been previously demonstrated $[20,21]$, some authors have reported more recently that MR-CVCs may be prone to colonization by this yeast [22-24].

Therefore, the aim of this manuscript is to compare and clarify the efficacy of the recommended CSS and MR-impregnated CVCs against the most frequent yeasts isolated from CLABSIs, in order to help clinicians to make the best option for patients at high risk of invasive Candida infection, whenever other preventive strategies have failed.

\section{Methods}

\section{Strains}

Type strains belonging to the American Type Culture Collection (ATCC) or to the Centraalbureau voor Schimmelcultures (CBS) were used, along with clinical isolates of C. albicans, C. glabrata and C. parapsilosis sensu stricto previously collected from patients admitted at a tertiary university hospital and identified using the Vitek 2 system (bioMérieux, France) (Table 1).

Yeast strains were kept frozen in yeast peptone dextrose medium (YPD) (Formedium, Hunstanton, England) supplemented with $40 \%$ glycerol at $-70{ }^{\circ} \mathrm{C}$ until testing. For each experiment, microorganisms were subcultured twice
Table 1 Microbial strains used: distribution by species and provenance

\begin{tabular}{lll}
\hline Strain & Isolate & Site of isolation or Source \\
\hline ATCC 90028 & C. albicans & American Type Culture Collection \\
CA075 & C. albicans & Central venous catheter \\
ATCC 22019 & C. parapsilosis & American Type Culture Collection \\
CP007 & C. parapsilosis & Central venous catheter \\
CBS138 & C. glabrata & CBS Collection \\
CG044 & C. glabrata & Central venous catheter \\
\hline
\end{tabular}

on Sabouraud agar (Liofilchem, Italy), at $35{ }^{\circ} \mathrm{C}$, during $24 \mathrm{~h}$ to assess the purity of the culture and its viability.

\section{Biofilm formation}

\section{Medium and growth conditions}

Candida strains were grown overnight in Sabouraud broth at $37{ }^{\circ} \mathrm{C}$ and $180 \mathrm{rpm}$. Afterwards, yeast cells were harvested by centrifugation, washed with PBS and standardized to $1 \times 10^{6}$ cells $/ \mathrm{mL}$ in Roswell Park Memorial Institute (RPMI) 1640 medium supplemented with L-glutamine and buffered with MOPS acid (Sigma-Aldrich).

\section{Biofilm formation assays}

Three different central venous catheters were used as substrate for the biofilm formation assays: standard uncoated polyurethane double-lumen central venous catheters (Arrow ${ }^{\circ}$ International, Inc. Reading, PA, USA), minocycline-rifampin impregnated central venous catheters (Cook $^{\circ}$ Medical, Bloomington, Inc., USA) and second-generation chlorhexidine/silver sulfadiazineimpregnated central venous catheters (Arrowgard Blue Plus, Arrow ${ }^{\circ}$ International, Inc., Reading, PA, USA). For biofilm assays, $1-\mathrm{cm}$ segments of each catheter were used. For each experimental condition, 3 catheter segments were used and experiments were performed twice, in different days.

To evaluate biofilm formation in the catheters, $2 \mathrm{~mL}$ of standardized yeast suspensions $\left(1 \times 10^{6}\right.$ cells $\left./ \mathrm{mL}\right)$ [25] were added to glass vials containing one segment of each catheter and allowed to form biofilm for 24, 48 and $72 \mathrm{~h}$, at $37^{\circ} \mathrm{C}$, with agitation (180 rpm). At 24 and $48 \mathrm{~h}$ time points, the catheters were washed with PBS and placed in a new tube with $2 \mathrm{~mL}$ of fresh RPMI. Catheters incubated only with RPMI were used as negative controls. For each time point, catheters were washed with PBS and the biofilm metabolic activity was quantified using the semi-quantitative XTT reduction assay [25]. Briefly, $2 \mathrm{ml}$ of a XTT/menadione solution $(0.5 \mathrm{~g} / \mathrm{L}$ and $1 \mu \mathrm{M}$ respectively) were added to each catheter and incubated in the dark for $3 \mathrm{~h}$ at $37{ }^{\circ} \mathrm{C}$. Then the supernatant absorbances were read in a spectrophotometer at $490 \mathrm{~nm}$. 


\section{Statistical analysis}

Biofilm formation, in different time points, was evaluated with One-Way ANOVA (with the Bonferroni correction. Statistical significance was considered as a $p$ value smaller than 0.05 . All statistical analysis was performed using the SPSS software (v. 20.0).

\section{Results}

\section{Biofilm formation on standard polyurethane central} venous catheters

C. glabrata was the species that showed higher values of biofilm metabolic activity at $24 \mathrm{~h}$ and $48 \mathrm{~h}$ time periods. At these time periods, C. albicans and C. parapsilosis exhibited similar values of biofilm metabolic activity (Fig. 1).

At the $72 \mathrm{~h}$ time point, all the species had produced biofilm with higher metabolic activity when compared with the 24 and $48 \mathrm{~h}$ periods. C. glabrata and C. parapsilosis exhibited the higher values of biofilm formation at this period.

Absorbance values for negative controls showed the absence of background or contamination (values equal to zero).

\section{Effect of antimicrobial catheters in biofilm formation}

In general, the CSS catheter segments were the most efficient in preventing biofilm formation by all Candida species (Fig. 2a). The MR catheter segments were not able to prevent biofilm formation by all these species, promoting its development in some cases.

Regarding C. albicans, the CSS catheter segments were effective in biofilm inhibition for all strains at all the studied time points. The inhibition ranged from $60 \%$ to 93\%. The MR catheter segments promoted C. albicans biofilm formation, at all tested conditions (Fig. 2a).
Concerning C. glabrata, the species that produced more biofilm, CSS catheter segments were also efficient in biofilm inhibition, for all strains and time points, ranging from 97 to $100 \%$ of inhibition (Fig. 2b). The MR catheters showed a slight reduction in the strain CBS 138 at 24 and $72 \mathrm{~h}$ time points and for strain CG044 at $48 \mathrm{~h}$ time point. However, reduction was never inferior to $50 \%$ of biofilm metabolic activity (Fig. $2 \mathrm{~b}$ ).

C. parapsilosis biofilm formation was also inhibited in a large extent by the CSS catheter segments (70 to $100 \%$ of biofilm inhibition), at all studied time points (Fig. 2c). The MR catheters were unable to prevent biofilm formation in this species, at all tested conditions (Fig. 2c).

Absorbance values for negative controls showed the absence of background or contamination (values equal to zero).

\section{Discussion}

CLABSIs remain a global medical concern and further work on preventive measures must definitely be pursued in order to reduce its incidence. Hence, when CLABSI rate is high, the $\mathrm{CDC}$ strongly recommends the implementation of a strategy that should include the education of healthcare personnel responsible for catheter insertion and maintenance, the use of maximal sterile barrier precautions and skin antisepsis using $>0.5 \%$ chlorhexidine preparation with alcohol during CVC insertion. Whenever after successful implementation of such comprehensive strategy the CLABSI rate is not decreasing, the CDC equally recommends the use of CSS or MR-impregnated CVCs in patients whose catheter is expected to remain in place $>5$ days, since such catheters can decrease the risk for CLABSI and thus result in lower hospital final costs [17].

Compared to standard non-coated catheters, firstgeneration CSS-CVCs (coated only on the external

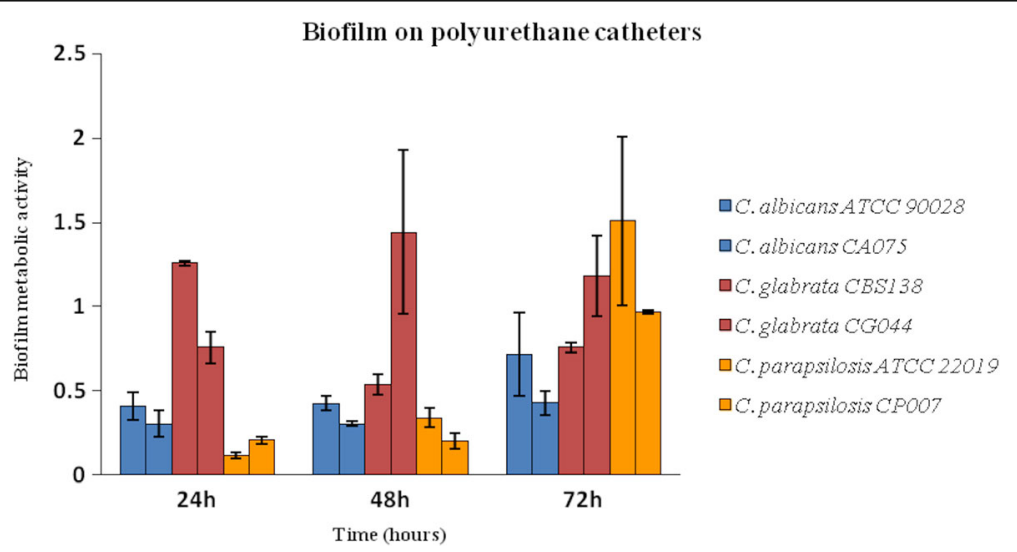

Fig. 1 Biofilm formation by different Candida species on standard uncoated polyurethane catheters at 24, 48 and $72 \mathrm{~h}$. Biofilms were quantified colorimetrically by XTT assay, which measures biofilm metabolic activity. Error bars represent the standard deviation among the results for different isolates. Each isolate was tested for its ability to form biofilm at least 6 times 

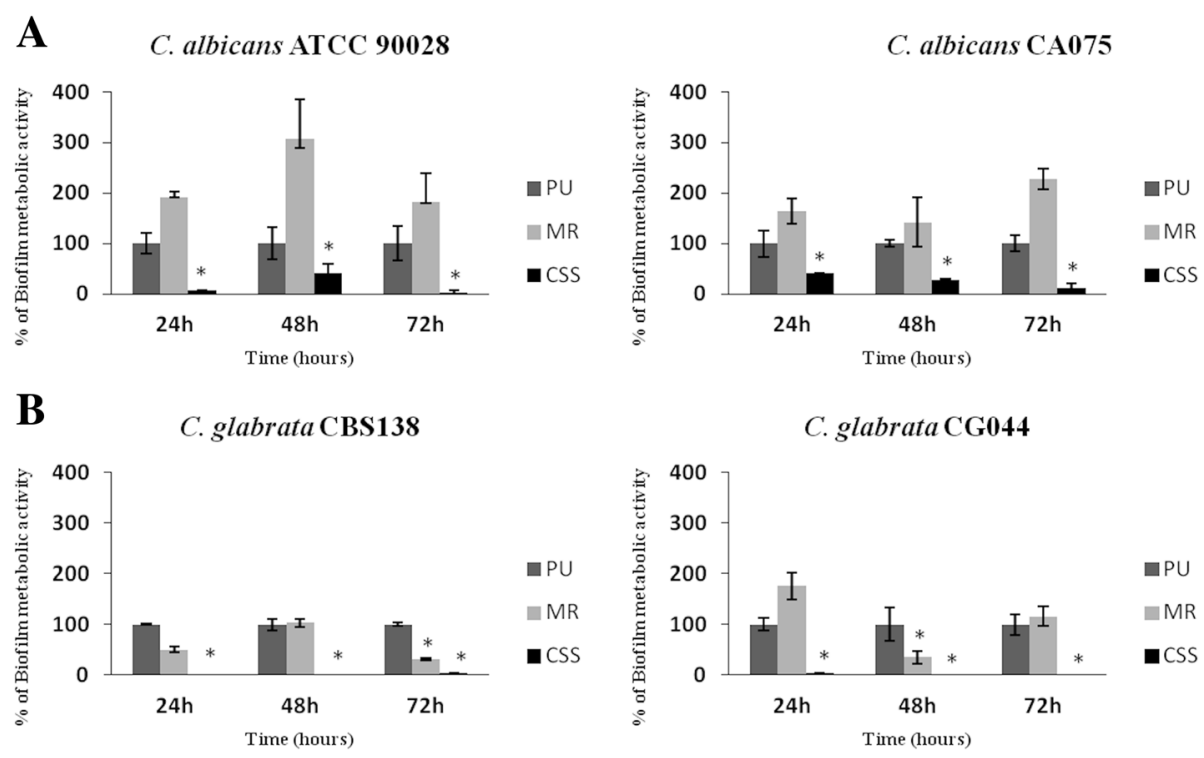

C

C. parapsilosis ATCC 22019

C. parapsilosis $\mathrm{CP007}$
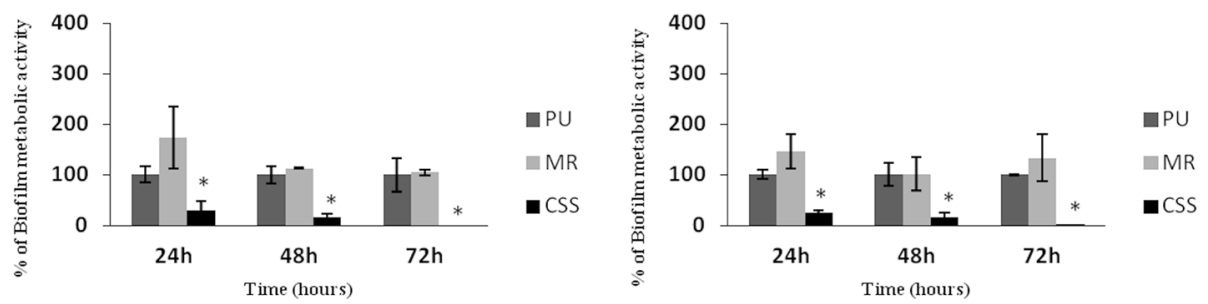

Fig. 2 In vitro biofilm formation on central venous catheters. Distinct Candida species were allowed to form biofilm for 24,48 and $72 \mathrm{~h}$ in segments of three different CVCs: standard uncoated polyurethane CVC (PU), minocycline-rifampin impregnated CVC (MR) and second-generation chlorhexidine/ silver sulfadiazine-impregnated (CSS). Graphics show the results for the two isolates of C. albicans (a), C. glabrata (b) and C. parapsilosis (c). Error bars represent the standard deviation among the results for different isolates. Each isolate was tested for its ability to form biofilm at least 6 times. * $P<0.05$ of coated catheters when compared with PU at each time point

luminal surface) did reduce the risk for CLABSI [26, 27]. Regarding the more recently available second-generation CVCs, a significant reduction in catheter colonization has been demonstrated, but a clear difference in CLABSI rate was not found due to low power of the randomized studies [28-30]. In the present biofilm formation experiment, CSS- impregnated CVCs effectively reduced biofilm formation by the most frequent yeast species causing CLABSIs, with inhibitions that ranged from $60 \%$ to $100 \%$. These strong inhibitory results certainly lack validation by clinical studies. The use of CSS-CVCs is not without concerns of hypersensitive reactions [31-33]. Even though, the CDC reports that the use of CSS-CVCs might be cost effective in ICU, burn and neutropenic patients and in other patient populations with rates of infection exceeding 3.3 per 1000 catheter days [34]. From a novel perspective, data from our experiment may further suggest that patients at high risk for invasive candidosis could benefit from the use of CSSimpregnated CVCs.
MR-CVCs (coated on both the internal and external luminal surfaces) were associated with lower rates of CLABSI when compared with first-generation CSSCVCs [35]. Since then, scarcer and less powered studies have been made comparing MR-CVCs with secondgeneration CSS-CVCs. Moreover, some controversy may still exist regarding the activity of MR-CVCs against yeasts: some authors have found a broad-spectrum activity against several bacteria and C. albicans $[20,36]$ and the technology sheet of the manufacturer of MR-CVCs reports activity against fungal infections. However, in our in vitro experiment, the MR-CVC segments were not only unable to prevent biofilm formation by $C$. albicans and C. parapsilosis strains, but also promoted biofilm formation by both yeasts, most markedly in the case of C. albicans. Regarding C. glabrata, the MR-CVC segments showed an inconspicuous effect, with a slight reduction in biofilm formation only at some time points. Using another in vitro model and considering C. albicans, Gaonkar et al. found similar results with increased 
adherence of the yeast to MR-CVCs compared to control CVCs [37]. Furthermore, on the meta-analysis of randomized controlled trials performed by Falagas et al., evidence was found that MR-impregnated catheters were prone to colonization with Candida [38]. Nevertheless, clinical trials have not found an increased incidence of CLABSIs due to Candida spp., even though the analyzed studies were underpowered to detect such difference. In vivo increased colonization of MR-CVCs by Candida spp. may result from the exposure of both surfaces of the catheter to a higher and continuous bacterial challenge from the hub and the insertion site [24]. Issues concerning the emergence of resistant pathogens with the use of MR-CVCs have been raised $[39,40]$, but still remain undocumented in the clinical setting [17].

In our study, a validated XTT reduction assay was used to evaluate the susceptibility of Candida biofilms when exposed to CSS and MR-impregnated CVCs [41]. One limitation of this assay that quantifies biofilm by colorimetric analysis is that it may not always correlate metabolic activities between different species [42]. However, this was not the case of our experiment, since the activity of CSS and MR-CVCs was compared using the same strains. Furthermore, in accordance with our results and using a different method (assessment of colony forming units with a modified Kirby-Bauer technique), Hanna et al. found not only that CSS-CVCs were superior against C. albicans and C. parapsilosis adherence, but also that the use of MR-CVC increased the colonization by Candida spp. [43].

In a meta-analysis conducted by Novikov et al. in hospitalized patients comparing the incidence of specific bacterial and fungal species colonizing antimicrobial CVCs and standard CVCs, it was found that antimicrobial CVCs in clinical use may become colonized with distinct microorganisms probably in relation to their antimicrobial spectrum of activity. In the particular case of Candida spp., the proportion of colonized MR-CVCs was greater than that of colonized standard CVCs [44]. Hence, antimicrobial CVCs may be advantageous over standard CVCs for specific microbial pathogens. As our experiment demonstrated, CSS-CVC exhibits an excellent activity against biofilm formation by the yeasts responsible for the majority of invasive candidosis; thus, patients at increased risk could well benefit from the use of such CSS-impregnated CVCs.

Instead of using clinical risk factors to tailor the selection of the best CVC for a given patient, alternatives are being studied targeting a wider antimicrobial spectrum per catheter. This is the case of the catheter impregnated with chlorhexidine (CHX) and MR, as proposed by Raad et al.: CHX-MR CVC may be more effective in completely inhibiting the biofilm formation by resistant bacteria and fungi, with prolonged antimicrobial activity [45].
Nevertheless, the implementation of this CVC would certainly benefit from further clinical experience and validation. Current $\mathrm{CDC}$ recommendations still contemplate nothing but two options: CSS and MRimpregnated CVCs. Another CVC impregnated with rifampicin-miconazole has been associated with a significantly lower risk of catheter colonization and CLABSIs compared to standard catheters in a prospective clinical trial in two university hospitals [46] and with a statistically significant reduction in the incidence of catheter-related bacteremia in patients with short-term catheter use at the central jugular and femoral sites in an ICU setting [47], but, again, further clinical validation and powered multicenter randomized studies are needed.

\section{Conclusions}

In this biofilm formation experiment, CSS-CVCs had a superior anti-Candida activity comparing to MR-CVCs. CSS-CVCs exhibited a strong antibiofilm activity against C. albicans and, moreover, against the emerging C. glabrata and C. parapsilosis. This study further documented not only the lack of antibiofilm activity of MR-CVCs against the tested yeasts, but also the promotion of biofilm formation in in vitro conditions, particularly of C. albicans. Therefore, following current CDC recommendations to use antimicrobial impregnated CVCs whenever CLABSI rate remains high and regarding patients at serious risk of invasive Candida infection, the use of CSS-CVCs may be suggested.

\section{Acknowledgments \\ Not applicable. \\ Funding \\ None to declare.}

Availability of data and materials

All the data supporting conclusions are available in Table 1 and Figs. 1 and 2.

Authors' contributions

All authors participated in the design of the work. All authors read and approved the final manuscript.

Ethics approval and consent to participate

Not applicable in this type of study.

Consent for publication

Not applicable.

\section{Competing interests}

The authors declare that they have no competing interests.

\section{Publisher's Note}

Springer Nature remains neutral with regard to jurisdictional claims in published maps and institutional affiliations.

\section{Author details}

${ }^{1}$ Division of Microbiology, Department of Pathology, Faculty of Medicine, University of Porto, Alameda Prof. Hernâni Monteiro, 4200 Porto, Portugal. ${ }^{2}$ Burn Unit, Department of Plastic and Reconstructive Surgery, Centro 
Hospitalar São João, Porto, Portugal. ${ }^{3}$ CINTESIS, Center for Health Technology and Services Research, Faculty of Medicine, University of Porto, Porto, Portugal.

Received: 1 September 2017 Accepted: 25 October 2017 Published online: 03 November 2017

\section{References}

1. Centers for Disease Control and Prevention, Bloodstream infection event (Central Line-Associated Bloodstream Infection and non-central line-associated Bloodstream Infection), January 2017, https://www.cdc.gov/nhsn/pdfs/ pscmanual/4psc_clabscurrent.pdf.

2. CDC National and State Healthcare-Associated Infections Progress Report, March 2014, http://www.cdc.gov/HAl/progress-report/hai-progress-report.pdf.

3. ECDC Suveillance Report, Annual epidemiological report, Antimicrobia resistance and healthcare-associated infections, 2014, https://ecdc.europa. eu/sites/portal/files/media/en/publications/Publications/antimicrobialresistance-annual-epidemiological-report.pdf.

4. Magill SS, Edwards JR, Fridkin SK. Emerging infections program healthcareassociated I, antimicrobial use prevalence survey T. Survey of health careassociated infections. N Engl J Med. 2014;370:2542-3.

5. Raad I, Reitzel R, Jiang Y, Chemaly RF, Dvorak T, Hachem R. Anti-adherence activity and antimicrobial durability of anti-infective-coated catheters against multidrug-resistant bacteria. J Antimicrob Chemother. 2008;62:746-50.

6. Wenzel RP. Nosocomial Candidemia - risk-factors and attributable mortality. Clin Infect Dis. 1995:20:1531-4.

7. Donlan RM. Biofilms and device-associated infections. Emerg Infect Dis. 2001;7:277-81.

8. Ingham CJ, Boonstra S, Levels S, de Lange M, Meis JF, Schneeberger PM. Rapid Susceptibility Testing and Microcolony Analysis of Candida spp. Cultured and Imaged on Porous Aluminum Oxide. Plos One. 2012;7:e33818.

9. Donlan RM. Role of biofilms in antimicrobial resistance. ASAIO J. 2000;46: S47-52.

10. Pfaller MA, Diekema DJ, Procop GW, Rinaldi MG. Multicenter comparison of the VITEK 2 antifungal susceptibility test with the CLSI broth microdilution reference method for testing amphotericin $B$, flucytosine, and voriconazole against Candida spp. J Clin Microbiol. 2007:45:3522-8.

11. Pfaller MA, Moet GJ, Messer SA, Jones RN, Castanheira M. Candida bloodstream infections: comparison of species distributions and antifungal resistance patterns in community-onset and Nosocomial isolates in the SENTRY antimicrobial surveillance program, 2008-2009. Antimicrob Agents Ch. 2011;55:561-6.

12. Wey SB, Mori M, Pfaller MA, Woolson RF, Wenzel RP. Risk factors for hospitalacquired candidemia. A matched case-control study. Arch Intern Med. 1989; 149:2349-53.

13. Sydnor ER, Perl TM. Hospital epidemiology and infection control in acute-care settings. Clin Microbiol Rev. 2011;24:141-73.

14. Horn DL, Neofytos D, Anaissie EJ, Fishman JA, Steinbach WJ, Olyaei AJ, et al. Epidemiology and outcomes of Candidemia in 2019 patients: data from the prospective antifungal therapy alliance registry. Clin Infect Dis. 2009;48: 1695-703.

15. Sardi JCO, Scorzoni L, Bernardi T, Fusco-Almeida AM, Giannini MJSM Candida species: current epidemiology, pathogenicity, biofilm formation, natural antifungal products and new therapeutic options. J Med Microbiol. 2013:62:10-24.

16. Pereira GH, Muller PR, Szeszs MW, Levin AS, Melhem MSC. Five-year evaluation of bloodstream yeast infections in a tertiary hospital: the predominance of non-C. Albicans Candida species. Med Mycol. 2010;48:839-42.

17. O'Grady NP, Alexander M, Burns LA, Dellinger EP, Garland J, Heard SO, et al. Guidelines for the prevention of intravascular catheter-related infections. Clin Infect Dis. 2011:52:e162-93.

18. Arrow ${ }^{\circledast}$ International. Arrowgard Blue ${ }^{\circledast}$ Antimicrobial Catheter Technology Information http://www.teleflex.com/usa/product-areas/vascular-access/ vascular -access-catheters/arrow-ergopack-system/safety-information/ arrowgard-blue-technology-information.pdf. Accessed 21 Aug 2017

19. Cook $^{\otimes}$ Medical. Spectrum ${ }^{\otimes}$ Minocycline/rifampin catheter. https://www. cookmedical.com/data/resources/6\%20CC-BM-ABRMCAT-EN-201202.pdf. Accessed 21 Aug 2017

20. Schierholz JM, Fleck C, Beuth J, Pulverer G. The antimicrobial efficacy of a new central venous catheter with long-term broad-spectrum activity. J Antimicrob Chemother. 2000;46:45-50.
21. AFE R, Guttler K, Konig DP, Yucel N, Korenkov M, Schierholz JM. Pharmacokinetics of the antimicrobial agents rifampicin and miconazole released from a loaded central venous catheter. J Hosp Infect. 2003;53:129-35.

22. Fraenkel D, Rickard C, Thomas P, Faoagali J, George N, Ware RA. Prospective, randomized trial of rifampicin-minocycline-coated and silver-platinum-carbonimpregnated central venous catheters. Crit Care Med. 2006;34:668-75.

23. Darouiche RO, Berger DH, Khardori N, Robertson CS, Wall MJ, Metzler MH, et al. Comparison of antimicrobial impregnation with tunneling of long-term central venous catheters - a randomized controlled trial. Ann Surg. 2005; 242:193-200.

24. Leon C, Ruiz-Santana S, Rello J, de la Torre MV, Valles J, Alvarez-Lerma F, et al. Benefits of minocycline and rifampin-impregnated central venous catheters. A prospective, randomized, double-blind, controlled, multicenter trial. Intensive Care Med. 2004;30:1891-9.

25. Pierce CG, Uppuluri P, Tristan AR, Wormley FL Jr, Mowat E, Ramage G, et al. A simple and reproducible 96-well plate-based method for the formation of fungal biofilms and its application to antifungal susceptibility testing. Nat Protoc. 2008;3:1494-500

26. Mermel LA. Prevention of intravascular catheter-related infections. Ann Intern Med. 2000;132:391-402.

27. Veenstra DL, Saint S, Saha S, Lumley T, Sullivan SD. Efficacy of antisepticimpregnated central venous catheters in preventing catheter-related bloodstream infection - a meta-analysis. Jama-J Am Med Assoc. 1999;281:261-7.

28. Brun-Buisson C, Doyon F, Sollet JP, Cochard JF, Cohen Y, Nitenberg G. Prevention of intravascular catheter-related infection with newer chlorhexidinesilver sulfadiazine-coated catheters: a randomized controlled trial. Intens Care Med. 2004:30:837-43.

29. Ostendorf T, Meinhold A, Harter C, Salwender H, Egerer G, Geiss HK, et al. Chlorhexidine and silver-sulfadiazine coated central venous catheters in haematological patients - a double-blind, randomised, prospective, controlled trial. Support Care Cancer. 2005;13:993-1000.

30. Rupp ME, Lisco SJ, Lipsett PA, Ped TM, Keating K, Civetta JM, et al. Effect of a second-generation venous catheter impregnated with chlorhexidine and silver sulfadiazine on central catheter - related infections - a randomized, controlled trial. Ann Intern Med. 2005;143:570-80.

31. Oda T, Hamasaki J, Kanda N, Mikami K. Anaphylactic shock induced by an antiseptic-coated central nervous catheter. Anesthesiology. 1997;87:1242-4.

32. Stephens $R$, Mythen M, Kallis P, Davies DWL, Egner W, Rickards A. Two episodes of life-threatening anaphylaxis in the same patient to a chlorhexidinesulphadiazine-coated central venous catheter. Brit J Anaesth. 2001;87:306-8.

33. Terazawa E, Shimonaka H, Nagase K, Masue T, Dohi S. Severe anaphylactic reaction due to a chlorhexidine-impregnated central venous catheter. Anesthesiology. 1998;89:1296-8.

34. Maki DG, Stolz SM, Wheeler S, Mermel LA. Prevention of central venous catheter-related bloodstream infection by use of an antisepticimpregnated catheter - a randomized, controlled trial. Ann Intern Med. 1997:127:257-66

35. Darouiche RO, Raad II, Heard SO, Thornby II, Wenker OC, Gabrielli A, et al. A comparison of two antimicrobial-impregnated central venous catheters. New Engl J Med. 1999;340:1-8.

36. Raad I, Darouiche R, Hachem R, Mansouri M, Bodey GP. The broad-spectrum activity and efficacy of catheters coated with minocycline and rifampin. J Infect Dis. 1996:173:418-24.

37. Gaonkar TA, Modak SM. Comparison of microbial adherence to antiseptic and antibiotic central venous catheters using a novel agar subcutaneous infection model. J Antimicrob Chemother. 2003;52:389-96.

38. Falagas ME, Fragoulis K, Bliziotis IA, Chatzinikolaou I. Rifampicin-impregnated central venous catheters: a meta-analysis of randomized controlled trials. Antimicrob Chemoth. 2007;59:359-69.

39. Tambe SM, Sampath L, Modak SM. In vitro evaluation of the risk of developing bacterial resistance to antiseptics and antibiotics used in medical devices. J Antimicrob Chemoth. 2001:47:589-98.

40. Sampath LA, Tambe SM, Modak SM. In vitro and in vivo efficacy of catheters impregnated with antiseptics or antibiotics: evaluation of the risk of bacterial resistance to the antimicrobials in the catheters. Infect Cont Hosp Ep. 2001:22:640-6.

41. Peeters $E$, Nelis HJ, Coenye T. Comparison of multiple methods for quantification of microbial biofilms grown in microtiter plates. J Microbiol Methods. 2008;72:157-65.

42. Chandra J, Mukherjee PK, Ghannoum MA. Vitro growth and analysis of Candida biofilms. Nat Protoc. 2008:3:1909-24. 
43. Hanna H, Bahna P, Reitzel R, Dvorak T, Chaiban G, Hachem R, et al. Comparative in vitro efficacies and antimicrobial durabilities of novel antimicrobial central venous catheters. Antimicrob Agents Ch. 2006;50: 3283-8.

44. Novikov A, Lam MY, Mermel LA, Casey AL, Elliott TS, Nightingale P. Impact of catheter antimicrobial coating on species-specific risk of catheter colonization: a meta-analysis. Antimicrob Resist In. 2012;1:40.

45. Raad I, Mohamed JA, Reitzel RA, Jiang Y, Raad S, Al Shuaibi M, et al. Improved antibiotic-impregnated catheters with extended-spectrum activity against resistant bacteria and fungi. Antimicrob Agents Chemother. 2012;56:935-41.

46. Yucel N, Lefering R, Maegele M, Max M, Rossaint R, Koch A, et al. Reduced colonization and infection with miconazole-rifampicin modified central venous catheters: a randomized controlled clinical trial. J Antimicrob Chemoth. 2004;54:1109-15.

47. Lorente L, Lecuona M, Ramos MJ, Jimenez A, Mora ML, Sierra A. The use of rifampicin-miconazole-impregnated catheters reduces the incidence of femoral and jugular catheter-related bacteremia. Clin Infect Dis. 2008;47: $1171-5$.

Submit your next manuscript to BioMed Central and we will help you at every step:

- We accept pre-submission inquiries

- Our selector tool helps you to find the most relevant journal

- We provide round the clock customer support

- Convenient online submission

- Thorough peer review

- Inclusion in PubMed and all major indexing services

- Maximum visibility for your research

Submit your manuscript at www.biomedcentral.com/submit
Biomed Central 